\title{
Quark-Lepton Universality and Large Leptonic Mixing
}

\author{
Anjan S. Joshipura ${ }^{1}$ and A. Yu. Smirnov ${ }^{2,3}$ \\ ${ }^{1}$ Theoretical Physics Group, Physical Research Laboratory, \\ Navrangpura, Ahmedabad 380 009, India \\ ${ }^{2}$ International Centre for Theoretical Physics, \\ Strada Costiera 11, 31014 Trieste, Italy \\ ${ }^{3}$ Institute for Nuclear Research, Russian Academy of Sciences, Moscow, Russia
}

\begin{abstract}
A unified description of fermionic mixing is proposed which assumes that in certain basis $(i)$ a single complex unitary matrix $V$ diagonalizes mass matrices of all fermions to the leading order, $(i i)$ the $S U(5)$ relation $M_{d}=M_{l}^{T}$ exists between the mass matrices of the down quarks and the charged leptons, and ( $i i i) M_{d}^{\dagger}=M_{d}$. These assumptions automatically lead to different mixing patterns for quarks and leptons: quarks remain unmixed to leading order $\left(i . e . V_{C K M}^{0}=1\right)$ while leptons have non-trivial mixing given by a symmetric unitary matrix $V_{P M N S}^{0}=V^{T} V . V$ depends on two physical mixing angles and for values of these angles $\sim 20^{\circ}-25^{\circ}$ it reproduces the observed mixing patterns rather well. We identify conditions under which the universal mixing $V$ follows from the universal mass matrices of fermions. Relatively small perturbations to the leading order structure lead to the CKM mixing and corrections to $V_{P M N S}^{0}$. We find that if the correction matrix equals the CKM matrix, the resulting lepton mixing agrees well with data and predicts $\sin \theta_{13}>0.08$. In a more general context, the assumption of partial universality, (i.e. different mixing for the up and the down components of doublets) is shown to lead to a complementarity relation $V_{P M N S} V_{C K M}=V^{T} V$ in the lowest order.
\end{abstract}




\section{Introduction}

Experimental results show striking differences in the mixing patterns of quarks and leptons: The quarks retain their flavour approximately and mix very little; leptons mix strongly; two of the three leptonic mixing angles are large and one of them may even be maximal. Understanding these differences among quarks and leptons could be a key step to the theory of fermion masses and this problem is extensively discussed now [1].

It may happen that quarks and leptons are fundamentally different: The large leptonic mixing or possible quasi-degenerate structure of the neutrino mass spectrum may be signals of some special symmetry operating in the leptonic sector alone. In particular, the maximal (or close to maximal) atmospheric mixing and vanishing (very small) $\sin \theta_{13}$ indicate presence of symmetry like the $\mu-\tau$ interchange symmetry [2]. Degeneracy of spectrum and inverse mass hierarchy may be considered as an evidence of the $L_{e}-L_{\mu}-L_{\tau}$ lepton number symmetry [3].

Alternatively, the leptonic world may not be special and there may be underlying quark-lepton symmetry or unification. There are different ways to reconcile the quarklepton unification with strongly different patterns of quark and lepton mixings. For example, the $S U(5)$ relation $[1]$

$$
M_{l}=M_{d}^{T}
$$

between the down quark and the charged lepton mass matrices can simultaneously accommodate small quark and large leptonic mixing angles if the matrices (1) have lopsided form $[4,5]$. In $S O(10)$ the $b-\tau$ unification [6] can be related to large leptonic mixing if neutrino masses dominantly come from interactions with the Higgs triplets [7].

The quark-lepton symmetry may take more general form than that stipulated in grand unified theories and attempts to understand fermionic mixing along these lines postulate some universal form for all fermionic mass matrices. The observed differences arise from small perturbations to this unified description. Two examples discussed in the literature are the democratic mass matrices [8] and singular structure with hierarchical matrix elements [9]. First two generations of fermions remain massless in these ansatz and therefore small perturbations can make large differences in mass hierarchies and furthermore the seesaw mechanism with nearly singular mass matrix of the RH neutrinos lead to enhancement of lepton mixing [9]. While strictly maximal mixing is difficult to reconcile in this picture, the large leptonic mixing can be reproduced in agreement with data.

The quark-lepton unification may have the form of quark-lepton complementarity [10, 11]: the data indicate that quark and lepton mixing angles (at least for the first and 
second generations) sum up to maximal mixing, $\theta_{12}^{l}=\pi / 4-\theta_{C}$, where $\theta_{C}$ is the Cabibbo angle. This last equality may imply that there is (i) some structure in the leptonic sector (only) which generates maximal (bi-maximal) mixing and (ii) the quark-lepton symmetry which propagates the CKM - mixing from quarks to leptons. In view of strong difference of the mass hierarchies in the quark and lepton sectors the later implies that the CKM mixing matrix weakly depends on the mass hierarchies.

The aim of this paper is to discuss an alternative form of quark-lepton universality. This form is strongly motivated by grand unified theories, particularly, eq. (1) and has the virtue that different patterns for quark and lepton mixing is built into this universal ansatz. It is assumed that in certain (universality) basis the relation between the basis states and the mass eigenstates of all fermions is determined by a universal (complex) $3 \times 3$ unitary matrix $V$ (or its conjugate). In addition, if eq. (1) is also imposed then quarks and leptons are automatically distinguished: The CKM matrix is unity: $V_{C K M}=V^{\dagger} V$, while its leptonic analogue - the PMNS matrix [12] $V_{P M N S}=V^{T} V$ - contains two non-trivial mixing angles. These angles are related to the structure of $V$ and can assume large values even when angles specifying the basic mixing matrix $V$ are relatively small, of the order of the Cabibbo angle. The corrections to basic ansatz are required in order to generate a non-trivial $V_{C K M}$. The same (or similar) corrections also modify $V_{P M N S}$, so that a kind of complementarity relation appears in which quarks and lepton mixing angles sum up to the angles of $V^{T} V$ instead to the maximal ones as in [10].

We discuss our ansatz in the next section. Properties of the zero order mixing are described subsequently in section 3 and 4 . Section 5 contains analysis of possible corrections to these ansatz assuming that departures from universality in the quark and lepton sectors are correlated. We consider a specific scheme of perturbation in section 6 which leads to such departures. A possibility of embedding scenario into Grand Unification theory is outlined in section 7 . Some comments on this approach and summary are contained in section 8 .

\section{Ansatz}

Our ansatz is based on the relations among fermionic masses that follow in grand unified theories. The minimal version of $S U(5)$ implies relation (1) and a symmetric up quark mass matrix $M_{u}$. The Dirac neutrino mass matrix $M_{\nu D}$ gets related to $M_{u}$ in $S O(10)$ models - the minimal version giving

$$
M_{u}^{T}=M_{u}=M_{\nu D} .
$$


It is possible to obtain $S O(10)$ models which simultaneously satisfy equalities $(1,2)$. We first consider implications of the above mass relations for mixing and then make additional plausible assumptions to arrive at a universal mixing ansatz.

We assume that to leading order in some approximation, the upper (up quarks, neutrinos) and the lower (d quarks, charged leptons) components of all the weak doublets are diagonalized by (complex) unitary matrices $V^{\prime}$ and $V$ respectively. Specifically, assume

$$
\begin{gathered}
V^{\prime \dagger} M_{u}^{0} V^{*}=D_{u}^{0} \quad ; \quad V^{\prime \dagger} M_{\nu D}^{0} V^{\prime}=D_{\nu D}^{0} \\
V^{\dagger} M_{d}^{0} V=D_{d}^{0} \quad ; \quad V^{T} M_{l}^{0} V^{*}=D_{l}^{0}
\end{gathered}
$$

$D_{f}^{0}$ above refer to the diagonal mass matrices of fermions and superscript 0 is used to denote the leading order. The first of these relations is based on the $S O(10)$ equality (2) which in other way around follows from it if $D_{u}^{0}=D_{\nu D}^{0}$. Likewise, relation (1) follows from eq. (3) when $D_{d}^{0}=D_{l}^{0}$. An assumption of a hermitian $M_{d}$ is made here which does not appear in $S U(5)$ but it can be achieved by imposing the left-right symmetry in addition. The light Majorana neutrino mass matrix is assumed to be diagonalized in the same way as up-quarks:

$$
V^{\dagger} M_{\nu}^{0} V^{\prime *}=D_{\nu}^{0}
$$

The later can be reproduced by the seesaw mechanism if the Majorana mass matrix of the $\mathrm{RH}$ neutrinos $M_{R}$ is also diagonalized as $M_{\nu}^{0}$ :

$$
V^{\prime \dagger} M_{R}^{0} V^{\prime *}=D_{R}^{0}
$$

Then for light neutrinos we have (in the basis $\bar{\nu} \nu^{*}$ )

$$
M_{\nu}^{0}=-M_{D}^{0} M_{R}^{0^{-1}} M_{D}^{0 T}=-V^{\prime} D_{D}^{0} D_{R}^{0-1} D_{D}^{0} V^{\prime T}
$$

which satisfies eq.(4).

Eqs. $(3,4)$ imply that in the leading order the mixing matrix for quarks equals

$$
V_{C K M}^{0}=V^{\prime \dagger} V
$$

and for leptons

$$
V_{P M N S}^{0}=V^{T} V^{\prime}
$$

These equalities lead to the relation (apparently $\left.V^{\prime}=V V_{C K M}^{0 \dagger}\right)$ :

$$
V_{P M N S}^{0}=V^{T} V V_{C K M}^{0 \dagger}
$$


For complex $V, V^{T} V$ is non trivial with the result that the lepton and quark mixings can be different in spite of unifying relations among their mass matrices. From (9) we obtain

$$
V_{P M N S}^{0} V_{C K M}^{0}=V^{T} V
$$

which shows a kind of the quark-lepton complementarity: quark and lepton mixing angles add up to the angles of the $V^{T} V$ matrix. This complementarity differs from the one considered in the literature $[10,11]$ in which $V_{P M N S}^{0}$ is assumed to have the bi-maximal or tri-maximal [13] form and $V_{C K M}$ arises as a correction to it. It is not always possible to obtain such mixing in a natural way while the relation (9) follows naturally from the GUT relations supplemented with additional assumptions of hermitian $M_{d}$ and equality (5).

The smallness of the CKM mixing angles requires $V^{\prime} \sim V$. This suggests a universal ansatz in which $V^{\prime}$ is equated to $V$ to leading order in some approximation. This ansatz provides a universal description of all fermion mixings with the property that the leptonic mixing remains non-trivial, i.e., substituting $V^{\prime}=V$ in eq. (8), we obtain $V_{C K M}^{0}=$ $V^{\dagger} V=I$ and

$$
V_{P M N S}^{0}=V^{T} V
$$

Let us denote by $\tilde{f}$ the fermion basis which corresponds to the universal description. Then from eq. (3) we obtain that $\tilde{f}$ are related to the mass basis as

$$
\tilde{d}_{L}=V d_{L} \quad ; \quad \tilde{l}_{L}=V^{*} l_{L} \quad ; \quad \tilde{f}_{L}=V f_{L}, \quad(f=u, \nu)
$$

Let us emphasize that the different transformation properties of the charged lepton (compared to down quarks) are responsible for generating a non-trivial PMNS matrix. We shall designate this ansatz as version I.

In an alternative version - to be called version II of the ansatz the neutrino transformation property is different compared to the up-quarks. This version is based on the unified mass formula in the minimal $S O(10)$ according to which $M_{l}$ and $M_{d}$ are also symmetric and are proportional to the up quark mass matrix. So, they are all diagonalized by the same matrix and thus $V=V^{\prime}$ :

$$
V^{\dagger} M_{f}^{0} V^{*}=D_{f}^{0}, \quad f=u, d, l .
$$

Also the Dirac mass matrix of neutrinos may have the same property as in (13). It is however assumed that the left handed neutrino components transform differently compared to eq. (12):

$$
\tilde{\nu}_{L}=V^{*} \nu_{L},
$$


so that eqs. $(13,14)$ now imply $V_{C K M}^{0}=V^{\dagger} V=I$, and

$$
V_{P M N S}^{0^{\prime}}=V^{\dagger} V^{*}
$$

In this version the PMNS matrix differs from the earlier version (eq. (11)) by complex conjugation.

The different transformation properties of the neutrino compared to the up-quark may arise from some special structure of $M_{R}$. However more appealing possibility is that the usual seesaw (type-I) just suppresses the neutrino Dirac mass and the main contribution to the neutrino mass matrix comes from the type-II seesaw [7] with

$$
V^{T} M_{\nu}^{0} V=D_{\nu}^{0}
$$

Let us underline several points in connection with the proposed ansatz.

- The quark-lepton universality can be reconciled with difference of quark and lepton mixings. In contrast to previous proposals of the quark-lepton universality [8, 9] this difference appears already in the lowest order.

- The ansatz (12) distinguishes between the charged leptons and other fermions if $V$ is complex. The GUT relation (1) provides a basis of introducing this distinction which results in different mixing patterns between quarks and leptons.

- Equation (1) is known [1,4] to lead to different patterns of quark and lepton mixing if $M_{d}^{0}$ has non-symmetric and lopsided form. The lopsided form is not required in the present ansatz and hermitian $M_{d}^{0}$ as implied by eq. (3) is sufficient to produce the differences in quark and leptonic mixings.

- In the ansatz II special form of transformations of neutrinos can be related to different origin (e.g. see-saw type II) and smallness of neutrino masses.

- Mixing among fermions is universal but their mass matrices need not be so unless all $D_{f}^{0}$ are proportional and $V$ is real. In view of the strong hierarchy in masses, it is natural to assume that only the third generation is massive in which case, $M_{f}^{0}$ for all $f$ can be related to a universal matrix as we will show.

- The ansatz remains unspecified until $V$ is given. If $V$ were to describe only quark mixing then different choices of $V$ would be equivalent at the leading order. But due to eqs. (12,14), $V$ acquires a physical content and gets related to $V_{P M N S}$. This allows direct experimental determination of the universal basis unlike other universality ansatzs $[8,9]$ proposed before. 
Since $V_{C K M}=I$ to the lowest order, some additional sources of fermion masses should exist. In general, they will also contribute to lepton mixing. We assume that this additional mixing is small - of the order of the Cabibbo mixing, so that $V_{P M N S}^{0}=V^{T} V$ can be considered as the lepton mixing in the lowest order approximation.

\section{Properties of the zero order mixing matrix}

Let us explore properties of the mixing matrix $V_{P M N S}^{0} \equiv V^{T} V$. The results are applicable to both versions of the universality. Apparently $V_{P M N S}^{0}$ is a symmetric unitary matrix which can be parameterized ${ }^{1}$ as

$$
\begin{aligned}
V_{P M N S}^{0} & =P U P \\
U & =\left(\begin{array}{ccc}
c_{\theta} & -c_{\phi} s_{\theta} & -s_{\phi} s_{\theta} \\
-c_{\phi} s_{\theta} & e^{i \alpha} s_{\phi}^{2}-c_{\phi}^{2} c_{\theta} & -c_{\phi} s_{\phi}\left(c_{\theta}+e^{i \alpha}\right) \\
-s_{\phi} s_{\theta} & -c_{\phi} s_{\phi}\left(c_{\theta}+e^{i \alpha}\right) & e^{i \alpha} c_{\phi}^{2}-c_{\theta} s_{\phi}^{2}
\end{array}\right)
\end{aligned}
$$

where $c_{\theta} \equiv \cos \theta, c_{\phi} \equiv \cos \phi$, etc., $\theta, \phi$ and $\alpha$ are new free parameters. $P$ is a phase matrix:

$$
P=\operatorname{diagonal}\left(e^{i \rho}, e^{i \sigma}, 1\right) .
$$

The matrix $P$ on the left hand side of $U$ in eq. (17) can be rotated away by redefining the charged lepton fields. The one on the right hand side contains the Majorana phases associated with the neutrino fields.

The $V_{P M N S}^{0}$ depends on two instead of three mixing angles which implies restrictions on the physical parameters. Comparing eq.(17) with mixing matrix in the standard parameterization [1] we obtain the following expressions for the standard mixing angles:

$$
\begin{aligned}
\tan \theta_{12} & =-\cos \phi \tan \theta \\
\sin \theta_{13} & =-\sin \phi \sin \theta \\
\sin \theta_{23} & =-\frac{\sin \phi \cos \phi\left(\cos \theta+e^{i \alpha}\right)}{\sqrt{1-\sin ^{2} \phi \sin ^{2} \theta}} .
\end{aligned}
$$

Using these relations we find lines of constant values of the observables in the $\phi-\theta$ plane which are shown in Fig. 1. Notice that only $\theta_{23}$ depends on the phase $\alpha$. The Fig. 1 corresponds to $\alpha=0$. With increase in $\alpha$ the angle $\theta_{23}$ decreases and therefore the corresponding iso-contours shift to larger $\phi$ and $\theta$.

\footnotetext{
${ }^{1}$ The above parameterization was used in [14] to describe mass matrix for the degenerate neutrinos. Here it describes mixing matrix. Our choice differs from the one in [14] by an interchange $\theta \rightarrow-\theta$.
} 


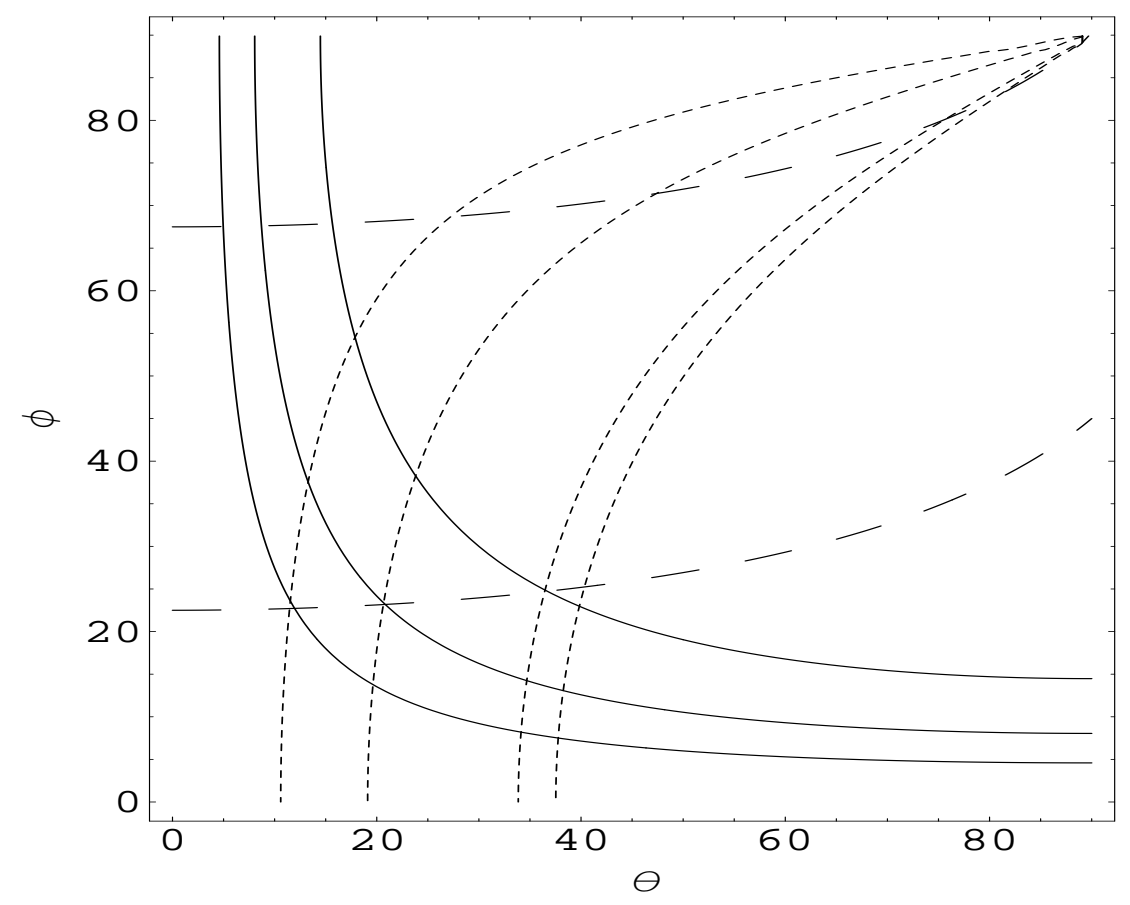

Figure 1: The contours of constant values of neutrino oscillation parameters in the $\phi-\theta$ plane for a symmetric PMNS matrix. The dotted curves correspond to $\theta_{12}=$ $10^{\circ}$ (left most) $19^{\circ}, 34^{\circ}, 37.5^{\circ}$. The dashed curves correspond to $\sin ^{2} 2 \theta_{23}=1.0$ and the solid ones - to $\sin \theta_{13}=0.08$ (left most), $0.14,0.25$.

An important consequence of the restricted form for $V_{P M N S}^{0}$ is that it can not reproduce the bi-maximal (as well as tri-bimaximal [13]) mixing matrix which implies zero 1-3 mixing. Indeed, according to (19), the condition $\sin \theta_{13}=0$ requires that either the solar or the atmospheric angle vanishes. Conversely, $\theta_{12}=\theta_{23}=45^{\circ}$ lead to unacceptable value $\sin \theta_{13} \approx 0.7$ unless some other sources of masses and mixing compensate this zero order mixing.

$V_{P M N S}^{0}$ can, however, provide a good zeroth order approximation. In particular, it can be a source of single maximal mixing. For maximal 2-3 mixing we find the isoline $\tan \theta_{23}=-1$ :

$$
\cos \theta=\frac{1-\tan \phi}{\tan \phi(1+\tan \phi)}
$$

The central measured values $\theta_{12}=34^{\circ}$ and $\theta_{23}=45^{\circ}$ can be achieved at $\phi=25^{\circ}$ and $\theta=37^{\circ}$. This gives $\sin \theta_{13}=0.25$ which is above the $3 \sigma$ allowed region. However corrections to the 1 - 3 mixing of the Cabibbo angle size, $\Delta \theta_{13} \sim \theta_{C}=13^{\circ}$ can easily lead to the acceptable values. The best fit isolines for $\theta_{12}$ and $\theta_{23}$ also cross in Fig. 1 at $\phi=76^{\circ}, \quad \theta=80^{\circ}$, which implies $\sin \theta_{13}=0.95$. The latter is too large to be properly 
corrected by the Cabibbo angle scale.

According to Fig. 1, the $1 \sigma$ acceptable values of $1-3$ mixing, $\sin \theta_{13}<0.14$, require (for maximal 2-3 mixing) that $\theta_{12} \leq 19^{\circ}$. So, additional contribution to the 1-2 mixing of the order $\theta_{C}$ from some other source can bring the 1-2 mixing angle back to the central measured value. Notice that for such a possibility $\theta \sim 21^{\circ}, \phi \sim 23^{\circ}$. Next generation of the experiments (Double CHOOZ [16], JPARC[17]) may improve the bound on 1-3 mixing down to $\sin \theta_{13}<0.08$ (90\% C.L.). In this case (see Fig. 1) the maximal 2-3 mixing would require $\theta_{12} \leq 10^{\circ}$ which is not possible to correct by the Cabibbo-type rotation.

For non-zero $\theta_{12}$ and $\theta_{23}$ the lower bound on $\sin \theta_{13}$ appears which can be quantified in the following way. According to eqs. $(17,19)$

$$
\begin{aligned}
\left|\sin \theta_{13}\right| & \geq \frac{\left|V_{\mu 3}\right|}{\left|V_{e 2}\right|}\left(1-\left|V_{e 1}\right|\right) \\
& \geq \frac{\left|\sin \theta_{23}\right|}{\left|\sin \theta_{12}\right|}\left(1-\left|V_{e 1}\right|\right) \geq \frac{\left|\sin \theta_{23}\right|}{\left|\sin \theta_{12}\right|}\left(1-\left|\cos \theta_{12}\right|\right),
\end{aligned}
$$

where equality holds if $\alpha=0$. Here $V_{\alpha i}$ are the elements of the PMNS matrix. For central values of the mixing angles, eq. (21) gives $\left|\sin \theta_{13}\right| \geq 0.23$. This bound weakens with decrease of $\theta_{12}$ and $\theta_{23}$. Taking the $3 \sigma$ lower values for these angles we find from eq. (21): $\left|\sin \theta_{13}\right| \geq 0.16$ which is at the $2 \sigma$ upper bound.

For non-zero $\alpha$ the 1-3 mixing equals

$$
\left|\sin \theta_{13}\right|=\frac{\left|V_{\mu 3}\right|}{\left|V_{e 2}\right|}\left(1-\mid V_{e 1}\right)\left[1-\frac{2 \cos \theta(1-\cos \alpha)}{(1+\cos \theta)^{2}}\right]^{-1 / 2} .
$$

One can see that expected value of $\sin \theta_{13}$ increases with $\alpha$, thus worsening the overall fit.

The matrix $V_{P M N S}^{0}$ alone (without corrections) does not reproduce well the observed lepton mixing matrix. The best global fit of the data would correspond to $(2-3) \sigma$ lower values of $\theta_{12}$ and $\theta_{23}$ and $\theta_{13}$ at $(2-3) \sigma$ upper bound. This fit corresponds to

$$
\phi \approx 19^{\circ}-25^{\circ} ; \quad \theta \approx 30^{\circ}-40^{\circ}, \quad \alpha=0
$$

which can be read-off from Fig. 1. Clearly region above the upper bound on $1-3$ mixing worsens the fit.

The neutrino masses that follow from the leading order expression, eq.(6) are immediately determined by the eigenvalues of the Dirac and Majorana mass matrices: $D_{\nu}^{0}=-D_{D}^{0} D_{R}^{0-1} D_{D}^{0}$. In particular, for the ratio we obtain:

$$
\frac{m_{\nu_{2}}^{0}}{m_{\nu_{3}}^{0}}=\left(\frac{m_{2 D}^{0}}{m_{3 D}^{0}}\right)^{2} \frac{M_{3}^{0}}{M_{2}^{0}}
$$




\section{Universal mixing and universal mass matrices.}

The phenomenological determination of $V_{P M N S}^{0}$ can be directly used to obtain $V$ and hence the fermion mass matrices in the lowest order in the universal basis. By definition, $V$ satisfies the equality

$$
V^{*} V_{P M N S}^{0} V^{\dagger}=I
$$

which means that $V$ can be determined from diagonalization of $V_{P M N S}^{0}$. In this way we find

$$
V=D^{*} \tilde{V} P
$$

where $P$ is given by eq. (18); the diagonal matrix $D$

$$
D=\operatorname{diag}(1, i, 1)
$$

plays crucial role in generation of the non-zero lepton mixing and

$$
\tilde{V}=\left(\begin{array}{ccc}
\cos \frac{\theta}{2} & -\cos \phi \sin \frac{\theta}{2} & -\sin \phi \sin \frac{\theta}{2} \\
-\sin \frac{\theta}{2} & -\cos \phi \cos \frac{\theta}{2} & -\sin \phi \cos \frac{\theta}{2} \\
0 & \sin \phi e^{i \alpha / 2} & -\cos \phi e^{i \alpha / 2}
\end{array}\right)
$$

It can be represented as $\tilde{V}=Z_{\alpha} R_{12}(\theta / 2) R_{23}(\phi) Z_{1}$, where $Z_{\alpha} \equiv \operatorname{diag}\left(1,1, e^{i \alpha / 2}\right), Z_{2} \equiv$ $\operatorname{diag}(1,-1,-1)$. The matrix $V$ is determined from $V_{P M N S}^{0}$ only up to an orthogonal transformations $O$, since $V$ and $O V$ both lead to the same $V_{P M N S}^{0}$ and unit $V_{C K M}$. All leading structures differing by $O$ are thus physically equivalent and we shall choose $O=1$.

Notice that the basic mixing angles appearing in eq. (28), $\phi \sim \theta / 2 \sim \mathcal{O}\left(15^{\circ}-20^{\circ}\right)$, are relatively small - not far away from the Cabibbo angle. Starting with such a small mixing, the above ansatz is able to reproduce the leptonic phenomenology reasonably well.

Using relations $(3-5)$ (with $V^{\prime}=V$ ) and expression for $V(26)$ we can write the mass matrices in the universal basis as

$$
\begin{aligned}
& M_{u p}^{0}=D^{*} \tilde{V} P^{2} D_{u p}^{0} \tilde{V}^{T} D^{*}, \quad(u p=u, \nu D, R), \\
& M_{d}^{0}=D^{*} \tilde{V} D_{d}^{0} \tilde{V}^{\dagger} D, \quad M_{l}^{0}=D \tilde{V}^{*} D_{l}^{0} \tilde{V}^{T} D^{*},
\end{aligned}
$$

for the version I and

$$
\begin{gathered}
M_{f}^{0}=D^{*} \tilde{V} P^{2} D_{f}^{0} \tilde{V}^{T} D^{*}, \quad(f=u, d, l), \\
M_{\nu}^{0}=D \tilde{V}^{*} P^{* 2} D_{\nu}^{0} \tilde{V}^{\dagger} D
\end{gathered}
$$

for the version II. 
Natural origin of the universality of mixing proposed in this paper would be universality of the mass matrices, at least in some approximation.

According to $(29-32), M_{f}^{0}$ for all fermions can be related to the same universal mass matrix in the approximation in which only the third generation fermions are massive, i.e. $D_{f}^{0}=\operatorname{diag}\left(0,0, m_{3 f}\right)$ and $\alpha=0$. (The later is required by phenomenology.) In this case, eqs. $(3,5)$ and (26) give

$$
M_{u}^{0}=m_{3 u} D^{*} \tilde{M} D^{*}, \quad M_{\nu}^{0}=m_{3 \nu} D^{*} \tilde{M} D^{*}, \quad M_{d}^{0}=m_{3 d} D^{*} \tilde{M} D, \quad M_{l}^{0}=m_{3 l} D \tilde{M} D^{*},
$$

in the first version, and

$$
M_{f}^{0}=m_{3 f} D^{*} \tilde{M} D^{*}, \quad(f=u, d, l), \quad M_{\nu}^{0}=m_{3 \nu} D \tilde{M} D
$$

in the second version. The universal matrix $\tilde{M}$ can be written as

$$
\tilde{M}=\left(\begin{array}{ccc}
s_{\phi}^{2} s_{\frac{\theta}{2}}^{2} & s_{\phi}^{2} s_{\frac{\theta}{2}} c_{\frac{\theta}{2}} & s_{\phi} c_{\phi} s_{\frac{\theta}{2}} \\
s_{\phi}^{2} s_{\frac{\theta}{2}} c_{\frac{\theta}{2}} & s_{\phi}^{2} c_{\frac{\theta}{2}}^{2} & s_{\phi} c_{\phi} c_{\frac{\theta}{2}} \\
s_{\phi} c_{\phi} s_{\frac{\theta}{2}} & s_{\phi} c_{\phi} c_{\frac{\theta}{2}} & c_{\phi}^{2}
\end{array}\right)
$$

$s_{\frac{\theta}{2}} \equiv \sin \frac{\theta}{2}, \quad c_{\frac{\theta}{2}} \equiv \cos \frac{\theta}{2}$.

All fermionic mass matrices are determined by a universal matrix $\tilde{M}$ as in the previous ansatz $[8,9]$. But unlike them, the presence of $D$ in eqs. $(33,34)$ changes the mixing patterns and allows large mixing for leptons even before perturbations are introduced.

If only partial universality is assumed: $V^{\prime} \neq V$, then from eq. (3) we find $\left(M_{u p}^{0}\right)_{i j}=$ $m_{3 u p} V_{i 3}^{\prime} V_{j 3}^{\prime}$. One can choose a parameterization for $V^{\prime}$ in such a way that $M_{u p}^{0}$ gets related to a matrix having the same form as in eq. (35) but with different mixing angles.

We can further absorb the complex matrices $D$ by the redefinition of fields:

$$
\tilde{f}=D^{*} f^{\prime}\left(f=u_{L}, u_{L}^{c}, d_{L}, d_{L}^{c}, \nu_{L}\right), \quad \tilde{l}_{L, R}=D l_{L, R}^{\prime}
$$

In the basis $f^{\prime}, l^{\prime}$, all the matrices equal $\tilde{M}$. Notice that in the new basis the charge current for the second generation of leptons has an opposite sign with respect to all other currents.

Since the universal matrices are singular (rank 1) this scenario can not be realized in the context of the see-saw with the same universal structure of $M_{R}$. One needs to assume that $M_{R}$ is non-singular but is still diagonalized by the same rotation as $M_{\nu D}$.

Let us consider the structure of the universal matrix $\tilde{M}$. Define $\epsilon_{1} \equiv \sin \phi$ and $\epsilon_{2} \equiv$ $\sin \frac{\theta}{2}$. According to eq. (23) both $\epsilon_{1}$ and $\epsilon_{2}$ are relatively small parameters, $0.3 \leq \epsilon_{1} \leq 0.4$ and $0.25 \leq \epsilon_{2} \leq 0.34$. Then $\tilde{M}$ assumes the following form if only the leading terms in 
$\epsilon_{1}, \epsilon_{2}$ are kept:

$$
\tilde{M}=\left(\begin{array}{ccc}
\epsilon_{1}^{2} \epsilon_{2}^{2} & \epsilon_{1}^{2} \epsilon_{2} & \epsilon_{1} \epsilon_{2} \\
\epsilon_{1}^{2} \epsilon_{2} & \epsilon_{1}^{2} & \epsilon_{1} \\
\epsilon_{1} \epsilon_{2} & \epsilon_{1} & 1
\end{array}\right)
$$

An ansatz very similar to eq. (37) was proposed in [9] for all fermion masses. The $\tilde{M}$ in eq. (37) coincides with the ansatz in [9] if one identifies $\epsilon_{1}$ and $\epsilon_{2}$ with the parameter $\lambda$ of [9]. The phenomenologically required values for $\epsilon_{1}, \epsilon_{2}$ are not very different from the value $\lambda \sim 0.26$ used in [9]. In the present case, the above form for $M_{f}^{0}$ arises from the basic ansatz and $V_{P M N S}^{0}$ determines $\epsilon_{1}, \epsilon_{2}$.

The specific form in eq. (37) has interesting consequences which were elaborated in [9]. One naturally gets hierarchical masses and the correct hierarchy in the quark mixing angles when small perturbations are added to this ansatz. We shall come back to these in sect. 6 .

Another possibility to introduce the universal mass matrix is to assume that in the lowest approximation $P^{2}=I$, and for all fermions the diagonal mass matrices have the same hierarchy of the eigenvalues: $D_{0} \equiv \operatorname{diag}\left(m_{1}, m_{2}, m_{3}\right)$. Then the universal matrix equals $\tilde{M}=\tilde{V} D_{0} \tilde{V}^{T}$. In this case the RH neutrino mass matrix can also have the universal form.

Two different universal diagonal matrices for up and down particles $D_{u p}^{0}$ and $D_{\text {down }}^{0}$ can be introduced in the context of partial universality, eq. (3).

\section{Corrections to $M_{f}^{0}$ and mixing}

The above ansatz needs corrections to account for the non-trivial quark mixing. Similar corrections to the leptonic sector would change the PMNS matrix as well. These two corrections may be independent or may also show some quark-lepton symmetry thus leading to kind of complementarity related to the zero order mixing $V_{P M N S}^{0}$. Notice that in [10] a bi-maximal form for $V_{P M N S}^{0}$ was assumed which leads to numerical relations between the solar and the Cabibbo angle such as $\theta_{\odot}=\pi / 4-\theta_{C}$. In the present case, the quark and lepton mixings differ by the angles of the zero order matrix.

Let us add a perturbation $\delta M_{f}$ to $M_{f}^{0}$ and consider

$$
M_{f}=M_{f}^{0}+\delta M_{f}
$$

$\delta M_{f}$ would lead to additional mixing $V_{f}$. Referring to the first scenario (sect. 2) we define,

$$
V_{f}^{\dagger} V^{\dagger} M_{f} V^{*} V_{f}^{*}=D_{f} \quad f=u, \nu D, R,
$$




$$
\begin{aligned}
V_{d}^{\dagger} V^{\dagger} M_{d} V V_{d} & =D_{d}, \\
V_{l}^{T} V^{T} M_{l} V^{*} V_{l}^{*} & =D_{l},
\end{aligned}
$$

where $D_{f}$ are the diagonal mass matrices after corrections. For simplicity, we have assumed $\delta M_{f}$ to have the same symmetry properties as $M_{f}^{0}$, i.e., symmetric for $f=u, \nu D, R$ and hermitian for $f=d, l$. Eq. (6) now gets replaced by

$$
\begin{aligned}
\mathcal{M}_{\nu} & =-M_{\nu D} M_{R}^{-1} M_{\nu D}^{T}, \\
& =-V V_{\nu D} \mathcal{F} D_{\nu} \mathcal{F}^{T} V_{\nu D}^{T} V^{T}
\end{aligned}
$$

where $\mathcal{F}$ satisfies

$$
\mathcal{F} D_{\nu} \mathcal{F}^{T}=D_{\nu D}\left(V_{\nu D}^{T} V_{R}^{*}\right) D_{R}^{-1}\left(V_{\nu D}^{T} V_{R}^{*}\right)^{T} D_{\nu D}
$$

and $D_{\nu}$ is a diagonal matrix of the light neutrino masses. It follows from the above that

$$
V_{\nu}=V V_{\nu D} \mathcal{F}
$$

This together with eq. (39) give us

$$
\begin{aligned}
V_{C K M} & =V_{u}^{\dagger} V_{d}, \\
V_{P M N S} & =V_{l}^{T} V_{P M N S}^{0} V_{\nu D} \mathcal{F} .
\end{aligned}
$$

The $V_{C K M}$ and corrections to $V_{P M N S}^{0}$ depend upon the perturbations. One can assume that they have similar origin and turn out to be of the same order.

Here we explore phenomenological consequences of assumption that the leading corrections to mixing display a quark-lepton symmetry.

Consider, e.g., the following simplest possibility:

(i) The CKM matrix arises essentially from corrections to the down quark matrix: $V_{d} \approx$ $V_{C K M}$ and $V_{u} \approx 1$.

(ii) The charged lepton and the Dirac neutrino mixing matrices satisfy $V_{d} \approx V_{l}^{*}$ (which corresponds to the zero order relation) and $V_{\nu D}=V_{u} \approx 1$.

(iii) $M_{R}$ receives very small corrections, so that $V_{R} \sim 1$.

The above assumptions imply $\mathcal{F} \approx 1$ in eq. (41) and the following relation

$$
V_{P M N S}=V_{C K M}^{\dagger} V_{P M N S}^{0} .
$$

The $V_{P M N S}$ is no longer a symmetric matrix because of the presence of the CKM matrix on the left. Also, the matrix $P$ on the left of eq.(17) now cannot be absorbed directly in redefining the charged lepton fields and can be physically relevant. The results presented below assume $P=1$. 
The eq. (43) can be rewritten as $V_{C K M} V_{P M N S}=V_{P M N S}^{0}$ showing a kind of complementarity of the quark and lepton mixings.

In the first approximation we can neglect 2-3 and 1-3 quark mixing considering $V_{C K M} \approx$ $V_{12}\left(\theta_{C}\right)$. We then obtain:

$$
V_{P M N S}=V_{12}^{\dagger}\left(\theta_{C}\right) U(\theta, \phi)
$$

where $U(\theta, \phi)$ is given in (17). The additional 1-2 rotation in (44) modifies all zero order mixing angles. It is easy to show that for large (nearly maximal) 2-3 mixing, this rotation simultaneously increases (or diminishes) $\theta_{12}^{0}$ and $\theta_{13}^{0}$. Due to smaller value $\theta_{13}^{0}$, the relative changes of the 1-3 mixing is stronger than 1-2 mixing. Therefore by $\sim \theta_{C}$ rotation one can improve fit of the data in comparison with zero order mixing by

1) taking larger values of $\phi$ and $\theta$ than in (23); this will lead to larger zero order values of $\theta_{12}^{0}$ and $\theta_{13}^{0}$;

2) performing $\theta_{C}$ rotation which reduces 1-2 and 1-3 mixing. Since reduction of the 1-3 mixing is stronger, we obtain smaller than in the previous case values of $\theta_{13}$ for the allowed values of $\theta_{12}$.

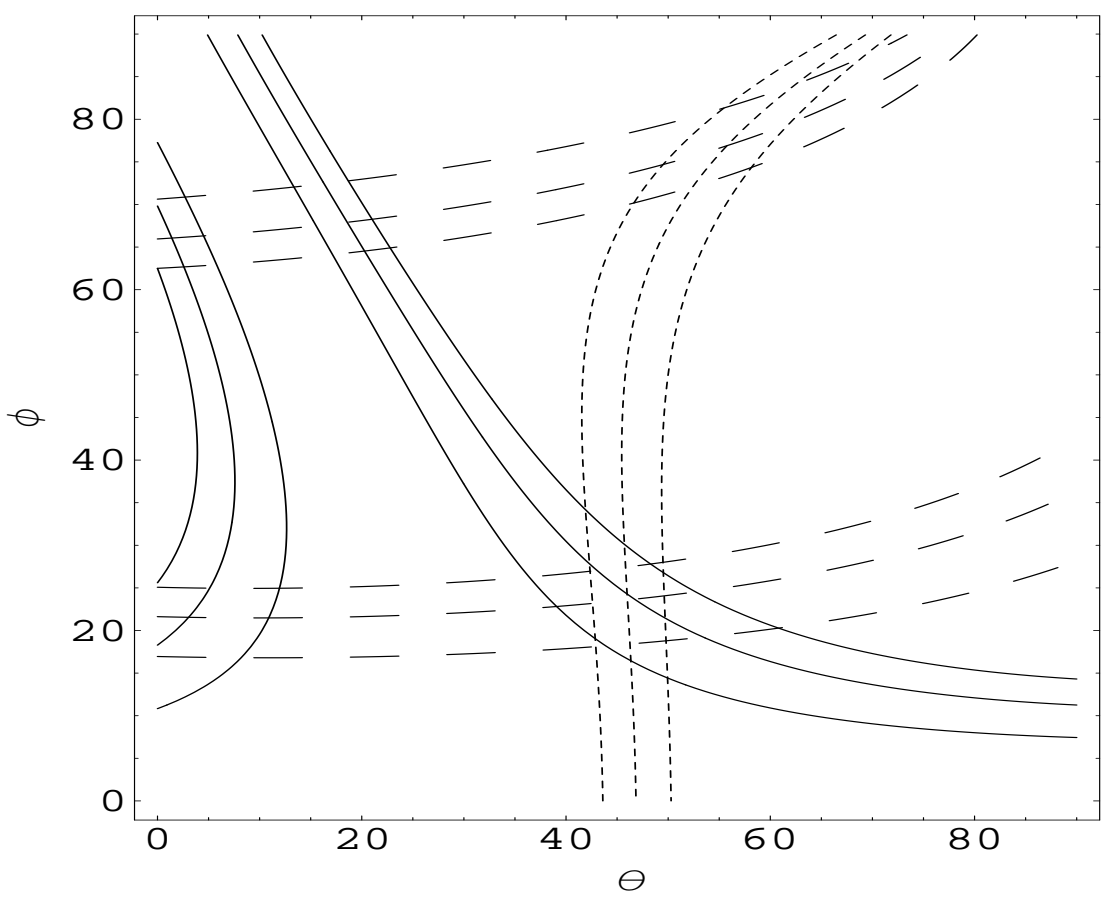

Figure 2: The contours of constant values of the neutrino oscillation parameters in the $\phi-\theta$ plane in the presence of the perturbations specified in the text. The dotted curves are for $\theta_{12}=30.6^{\circ}, 34^{\circ}, 37^{\circ}$. The dashed curves correspond to $\sin ^{2} \theta_{23}=0.34,0.5,0.62$ and the solid curves to $\sin \theta_{13}=0.09,0.14,0.17$. Central lines correspond to the best fit values and the outer ones restrict the $2 \sigma$ allowed regions. 
These features can be seen in Fig. 2, where we show $2 \sigma$ allowed ranges of observables in the $\phi-\theta$ plane. The full $V_{C K M}$ instead of $V_{12}\left(\theta_{C}\right)$ is used in drawing this figure. Unlike in the zeroth order case, now a sizable intersection region occurs at $2 \sigma$ which corresponds to

$$
\begin{gathered}
\phi \approx 20^{\circ}-28^{\circ}, \quad \quad \theta \approx 42^{\circ}-48^{\circ}, \\
\left|\sin \theta_{13}\right| \sim 0.09-0.17 .
\end{gathered}
$$

We find that at $3 \sigma$ level $\sin \theta_{13}$ still has a lower bound $\left|\sin \theta_{13}\right| \sim 0.08$ which can be tested in the next generation of experiments. The best fit values of observables $\tan ^{2} \theta_{12}=$ $0.45, \sin ^{2} 2 \theta_{23}=1$ and $\sin \theta_{13}=0.13$ are obtained for $\theta=46.01^{\circ}, \phi=23.6^{\circ}$.

In case of the partial universality, $V_{P M N S}$ is obtained by the right multiplication of $V^{T} V$ with $V_{C K M}^{\dagger}$, eq.(9). In this case, starting with initially negative $\tan \theta_{12}$, the $V_{12}^{\dagger}\left(\theta_{C}\right)$ rotation increases the value of $\tan ^{2} \theta_{12}$ without affecting $\theta_{13}$. As a consequence, one can obtain a good fit to data by initially choosing smaller values of $\phi, \theta$ corresponding to a smaller $\theta_{13}$ and $\theta_{12}$. Cabibbo rotation then increases the solar angle to the required value. Indeed, one finds (again using full $V_{C K M}$ ) that eq. (9) can reproduce the best fit values $\tan ^{2} \theta_{12}=0.45, \sin ^{2} \theta_{23}=0.5$, and predicts $\sin \theta_{13}=0.13$ with $\theta=22.35^{\circ}, \phi=24.34^{\circ}$.

\section{Universal mixing and mass hierarchies}

We now discuss specific perturbations to $M_{f}^{0}$ which approximately reproduce the scenario outlined above. Following [9], we assume that $\left(M_{f}^{0}\right)_{i j}$ receives sub-leading corrections parameterized by $\delta_{i j}^{f} \leq \mathcal{O}\left(\epsilon_{1}, \epsilon_{2}\right)$, and the $\tilde{M}$ in eq.(37) changes after perturbations to

$$
\tilde{M}_{f}=\left(\begin{array}{ccc}
\epsilon_{1}^{2} \epsilon_{2}^{2}\left(1+\delta_{11}^{f}\right) & \epsilon_{1}^{2} \epsilon_{2}\left(1+\delta_{12}^{f}\right) & \epsilon_{1} \epsilon_{2}\left(1+\delta_{13}^{f}\right) \\
\epsilon_{1}^{2} \epsilon_{2}\left(1+\delta_{12}^{f}\right) & \epsilon_{1}^{2}\left(1+\delta_{22}^{f}\right) & \epsilon_{1}\left(1+\delta_{23}^{f}\right) \\
\epsilon_{1} \epsilon_{2}\left(1+\delta_{13}^{f}\right) & \epsilon_{1}\left(1+\delta_{23}^{f}\right) & 1
\end{array}\right)
$$

Here $f$ refers to all fermions and $\delta_{i j}^{f}$ are taken to be real. These perturbations generate (i) masses for the first two generations, (ii) non-trivial CKM matrix, and (iii) corrections to $V_{P M N S}^{0}$. The ratios of masses are given by [9]

$$
\begin{aligned}
& \frac{m_{2}^{f}}{m_{3}^{f}} \approx \epsilon_{1}^{2}\left(\delta_{22}^{f}-2 \delta_{23}^{f}\right) \\
& \frac{m_{1}^{f}}{m_{3}^{f}} \approx \epsilon_{1}^{2} \epsilon_{2}^{2}\left(\delta_{11}^{f}-2 \delta_{13}^{f}-\frac{\left(\delta_{12}^{f}-\delta_{23}^{f}-\delta_{13}^{f}\right)^{2}}{\delta_{22}^{f}-2 \delta_{23}^{f}}\right) .
\end{aligned}
$$

The above relations hold for all fermions including the right handed neutrinos. 
The corrected $M_{f}$ is diagonalized as in eq.(39). The correction $V_{f}$ to the zeroth order matrix can be found by approximately diagonalizing $\tilde{M}_{f}$ through successive rotations [9]. This procedure leads to

$$
V_{f} \approx P^{*}\left(\begin{array}{ccc}
1 & -\epsilon_{2}\left(1-h^{f}\right) & -\epsilon_{1} \epsilon_{2}\left(\delta_{23}^{f}-\delta_{13}^{f}\right) \\
-\epsilon_{2}\left(1-h^{f}\right) & -1 & -\epsilon_{1} \delta_{23}^{f} \\
-\epsilon_{1} \epsilon_{2}\left(\delta_{23}^{f} h^{f}-\delta_{13}^{f}\right) & \epsilon_{1} \delta_{23}^{f} & -1
\end{array}\right)
$$

where

$$
h^{f}=\frac{\delta_{12}^{f}-\delta_{13}^{f}-\delta_{23}^{f}}{\delta_{22}^{f}-2 \delta_{23}^{f}}
$$

The mixing matrices follow from the eqs. (48) and (42). As noted in [9], the above $V_{d, u}$ automatically lead to hierarchical mixing angles for quarks, $V_{u s} \sim \epsilon, V_{c b} \sim \epsilon \delta, V_{u b} \sim \epsilon^{2} \delta$. As regards the leptonic sector, the basic mixing is still given by $\theta, \phi$ and perturbations generate additional corrections.

The main problem is to reconcile the proposed universality of mixing and strongly different mass hierarchies of quarks and leptons. Let us show that it is possible for particular choice of parameters $\delta$. We assume that perturbations to $M_{u}^{0}, M_{R}^{0}, M_{D}^{0}$ satisfy

$$
\delta_{12}^{f}=\delta_{22}^{f} \equiv \delta^{f} ; \quad \delta_{23}^{f} \approx \delta_{13}^{f} \approx 0
$$

In this case $m_{2}^{f} / m_{3}^{f} \approx \epsilon_{1}^{2} \delta^{f}$ and $h^{f}=1$. Consequently, effect of corrections is only to induce masses for the first two generations as in eq. (47). The mixing remains unchanged to leading order and is given by $V$ since $V_{f}$ (eq. (48)) has only diagonal elements when conditions (49) are imposed. Due to equality (49), correction to the 1-2 mixing appears in the higher order in $\epsilon$ :

$$
\Delta \theta_{12} \approx \epsilon_{2}^{3}\left(\frac{\delta_{11}^{f}}{\delta^{f}}-1\right) .
$$

If $\delta_{11}^{f}=\delta^{f}$ the correction is zero, but since $\delta^{f} \neq 0$ the mass of second eigenvalue can be changed arbitrary (within the application limits). This illustrates that certain symmetry (equalities) of corrections can lead to unchanged mixing but different hierarchies.

Perturbations to the down quark and the charged lepton mass matrices should generate the CKM mixing and therefore $\delta_{11,22}^{f}$ (for $f=d, l$ ) do not satisfy eq. (49). Freedom in these parameters is enough to reproduce the $V_{C K M}, V_{P M N S}$ and the mass hierarchies correctly. We have checked numerically that this can be done with corrections $\delta^{f} \leq 0.3$.

\section{Universality and Unification}

Our ansatz is based on the eqs. $(1,3,5)$. We now show that these relations can be obtained in an $S O(10)$ model with some assumptions. Specific model that we discuss gives eq.(1) 
and the following relations:

$$
M_{u}^{0}=M_{u}^{0 T}=-1 / 3 M_{\nu D}^{0}=\beta M_{R}^{0}
$$

where $\beta$ is the ratio of the weak scale to the right-handed neutrino mass scale. These equalities allow a single unitary matrix $V^{\prime}$ to simultaneously diagonalize $M_{u}^{0}, M_{\nu D}^{0}$ and $M_{R}^{0}$ as in eqs. $(3,5)$ and lead to eq. (10).

The eqs. $(1,51)$ arise from the following $S O(10)$ invariant couplings in the super potential:

$$
\begin{gathered}
W_{d}=\frac{f_{i j}}{M}\left(16_{i}^{T} H\right)\left(H^{\prime T} 16_{j}\right)+\frac{f_{i j}^{\prime}}{M}\left(16_{i}^{T} H^{\prime}\right)\left(H^{T} 16_{j}\right) \\
W_{u}=g_{i j} 16_{i}^{T} 16_{j} \Phi .
\end{gathered}
$$

Here, $H, H^{\prime}$ are 16-plets of Higgs; 16 refer to the fermions and $i, j$ are generation indices. $\Phi$ is a $\overline{126}$-plet. The multiplets in the parenthesis in eq.(52) are contracted to form $S O(10)$ vectors. Two remarks are in order.

- The superpotential (52) was used in [4] to obtain eq. (1). Since the 16-plet contains only the Higgs doublet $H_{d}$ with hypercharge $-1 / 2$, eq.(52) gives mass only to the charged leptons and the down quarks. The $H, H^{\prime}$ contain neutral fields with the quantum numbers of $\nu, \nu^{c}$. Vacuum expectation value (vev) for $\nu^{c}$ breaks $S O(10)$

preserving $\mathrm{SU}(5)$. This leads to $S U(5)$ relation eq. (1) when $\nu, \nu^{c}$ components of $H, H^{\prime}$ acquire vevs.

- $\overline{126}$ contains both the up type and the down type doublets, $H_{u, d}$, and triplets, $\Delta_{L, R}$ under respective $S U(2)$ groups. Vacuum expectation value for $H_{u}$ field generates eq. (51). One needs to assume here that $H_{d}$ component in $\overline{126}$ has zero vev at the minimum or that its contribution to $M_{d}, M_{l}$ is sub-dominant compared to the contribution from the superpotential (52).

This example demonstrates that differences in quark and lepton mixings can naturally arise in $S O(10)$ scheme. The mixing matrices satisfy the complementarity relation eq. (10) if $M_{d}$ is Hermitian. It is non-trivial to derive the relation $V^{\prime}=V$ used in the ansatz and may require a larger quark-lepton symmetry not envisaged in $S O(10)$.

\section{Discussion}

We discuss here some open questions. The proposed ansatz requires complete universality and treats the right handed neutrinos also on similar footings as other fermions in version 
I. Actually the right handed neutrino masses may have different origin and may not show complete universality. Some of the above considerations remain valid even if the right handed neutrinos display different mixing pattern, i.e.,

$$
\tilde{\nu}_{R}=\tilde{V}_{R} \nu_{R}
$$

This changes eq.(6) and $V_{P M N S}^{0}$ to

$$
\begin{gathered}
\mathcal{M}_{\nu}^{0}=-V D_{D}^{0} \hat{V} D_{R}^{0-1} \hat{V}^{T} D_{D}^{0} V^{T}, \\
V_{P M N S}^{0}=V^{T} V \mathcal{F}^{0}
\end{gathered}
$$

where $\hat{V}=V^{T} \tilde{V}_{R}$ is a general unitary matrix and $\mathcal{F}^{0}$ is defined as

$$
\mathcal{F}^{0 \dagger} D_{D}^{0} \hat{V} D_{R}^{0-1} \hat{V}^{T} D_{D}^{0} \mathcal{F}^{0 *}=D_{\nu}^{0}
$$

Also in this case, one does get different mixing matrices for quarks and leptons as required. The $V_{P M N S}^{0}$ is no longer strictly symmetric. Corrections due to $\mathcal{F}^{0}$ can be small due to hierarchy in masses $D_{D}^{0}$ if the diagonal elements of $\hat{V} D_{R}^{0}{ }^{-1} \hat{V}^{T}$ are not suppressed due to phases in $\hat{V}$. In the converse case, some large mixing angle may come from $\mathcal{F}^{0}$ and the restrictions on $\phi, \theta$ may get non-trivially modified.

The realization of the second version of our ansatz would require special neutrino structures and some additional symmetry to realize it. A simple possibility is using the type-II seesaw [7] in which case one requires eq. (16). The same version can be realized using type-I seesaw provided that $M_{R}$ has a specific structure. Assuming that all the Dirac mass matrices, including the Dirac mass matrix of neutrinos have the same universal structure as (13) we can determine $M_{R}$ to have a form

$$
\begin{aligned}
M_{R} & =V D_{D} V_{P M N S}^{0} D_{\nu}^{-1} V_{P M N S}^{0} D_{D} V^{T} \\
& =\left(V D_{D} V^{T}\right)\left(V D_{\nu}^{-1} V^{T}\right)\left(V D_{D} V^{T}\right)
\end{aligned}
$$

All the matrices should be non-singular here. Notice that $M_{R}$ turns out be product of three diagonal mass matrices all rotated through $V$. It would be interesting to look for special symmetries enforcing eq. (58) 
Before closing, let us recapitulate:

1). Motivated by the SU(5) relation between mass matrices of the charged leptons and down quarks we proposed a universal ansatz for the fermion mixing. According to this ansatz there exists certain (universality) basis in which mass matrices of all fermions but charge leptons (or neutrinos) are diagonalized by the same rotation matrix $V$ at the lowest order. The matrix for charged leptons (or neutrinos) is diagonalized by the conjugate $V^{*}$. This leads to an absence of quark mixing but non-trivial lepton mixing given by $V_{P M N S}^{0}=$ $V^{T} V$.

In this way we have realized an idea that difference in the quark and lepton mixing appears in some lowest approximation: the quark mixing is zero and the lepton mixing is non-trivial and, in general, large. The CKM mixing is realized as the correction.

2). We studied properties of the lowest order mixing matrix. $V_{P M N S}^{0}$ is symmetric matrix characterized by two physical angles $\theta$ and $\phi$. We showed that $V_{P M N S}^{0}$ is rather close to the observed mixing matrix for $\theta / 2 \approx \phi=20-25^{\circ}$. Furthermore, the generic feature of $V_{P M N S}^{0}$ is that the 1-3 mixing is close to the present upper bound.

3). The universal mixing matrix can follow from universal mass matrices of fermions. A natural realization is the one in which the mass matrices have only one (third) non zero eigenvalue in the lowest order.

4). We considered corrections to the lowest (universal) approximation which (a) generate

the CKM-mixing; (b) modify $V_{P M N S}^{0}$ leading to better agreement with observations, in particular, decrease $\sin \theta_{13}$.

Assuming (for simplicity) the quark - lepton universality, in which the correction matrix for lepton mixing equals (or similar to) the CKM matrix we obtain good agreement of $V_{P M N S}^{0}$ with data. In this case we predict $\sin \theta_{13}>0.08$ which can be tested in the next generation of experiments.

5). The suggested ansatz can be embedded into $\mathrm{SU}(5)$ and $\mathrm{SO}(10)$ GU models.

\section{References}

[1] R. N. Mohapatra et al., arXiv:hep-ph/0412099, hep-ph/0510213; G. Altarelli and F. Feruglio, Phys. Rept. 320, 295 (1999); New. J. of Phys. 6 (2004) 106 [hep- 
ph/0405048] J. W. F. Valle, Nucl. Phys. Proc. Suppl. 149, 3 (2005) [arXiv:hep$\mathrm{ph} / 0410103]$.

[2] T. Fukuyama and H. Nishura, hep-ph/9702253; R. N. Mohapatra and S. Nussinov, Phys. Rev. D 60013002 (1999); E. Ma and M. Raidal, Phys. Rev. Lett. 87 011802 (2001); C. S. Lam, Phys. Lett. B 507214 (2001); K.R.S. Balaji, W. Grimus and T. Schwetz, Phys. Lett. B 508301 (2001); Y. Koide, Phys. Rev. D 69 (2004) 093001; P. F. Harrison and W. G. Scott, Phys. Lett. B 547219 (2002); W. Grimus and L. Lavoura, Acta Phys. Polon. B 32, 3719 (2001) [arXiv:hep-ph/0110041]; JHEP 0107, 045 (2001) [arXiv:hep-ph/0105212]; E. Ma, Phys. Rev. D 66, 117301 (2002) [arXiv:hep-ph/0207352]; W. Grimus, A. S. Joshipura, S. Kaneko, L. Lavoura and M. Tanimoto, JHEP 0407, 078 (2004) [arXiv:hep-ph/0407112]; W. Grimus, S. Kaneko, L. Lavoura, H. Sawanaka, M. Tanimoto, arXiv:hep-ph/0510326; R. N. Mohapatra, JHEP 0410, 027 (2004); R. N. Mohapatra and W. Rodejohann, Phys. Rev. D 72053001 (2005); S. Choubey and W. Rodejohann, Eur. Phys. Journ. C 40 259 (2005); T. Kitabayashi and M. Yasue, Phys. Lett. B621 133 (2005)[arXiv:hepph/0504212], arXiv:hep-ph/0510132; A. Ghosal, Mod. Phys. Lett., A19, 2579 (2004).

[3] S. T. Petcov, Phys. Lett. B110 245 (1982). R. Barbieri et al, JHEP 9812017 (1998); A. S. Joshipura, Phys. Rev. D60, 053002 (1999) [arXiv:hep-ph/9808261]; A. S. Joshipura and S. D. Rindani, Eur. Phys. J. C 14, 85 (2000); ibid Phys. Lett. 464 239 (1999); R. N. Mohapatra, A. Perez-Lorenzana and C. A. de S. Pires, Phys. Lett. B474 355 (2000); T. Kitabayashi and M. Yasue, Phys. Rev. D 63095002 (2001); L. Lavoura and W. Grimus,JHEP 0009, 007 (2000) [arXiv:hep-ph/0008020]; H. S. Goh, R. N. Mohapatra and S. P. Ng, Phys. Lett. B 542, 116 (2002)[arXiv:hepph/0205131]; W. Grimus and L. Lavoura, J. Phys. G 31, 683 (2005)[arXiv:hepph/0410279];W. Grimus and L. Lavoura, Phys. Rev. D 62, 093012 (2000) [arXiv:hep$\mathrm{ph} / 0007011]$.

[4] C.H. Albright and S.M. Barr, Phys. Rev. D 58, 013002 (1998); C.H. Albright, K.S. Babu and S.M. Barr, Phys. Rev. Lett. 81, 1167 (1998); K. S. Babu and S. M. Barr, Phys. Lett. B 525, 289 (2002).

[5] G. Altarelli and F. Feruglio, Phys. Lett. B 451, 388 (1999). [arXiv: hep-ph/9812475].

[6] B. Bajc, G. Senjanovic and F. Vissani, Phys. Rev. Lett. 90, 051802 (2003) [arXiv:hepph/0210207]; H. S. Goh, R. N. Mohapatra and S. P. Ng, Phys. Lett. B 570, 215 (2003) [arXiv:hep-ph/0303055]. 
[7] R. N. Mohapatra and G. Senjanovic, Phys. Rev. D23 165 (1981); J. Schechter and J. W. F. Valle, Phys. Rev. D 222227 (1980); G. Lazarides, Q. Shafi and C. Wetterich, Nucl. Phys. B181 287 (1981).

[8] E. K. Akhmedov, G. C. Branco, F. R. Joaquim and J. I. Silva-Marcos, Phys. Lett. B 498, 237 (2001) [arXiv:hep-ph/0008010]; R. Dermisek, Phys. Rev. D 70, 073016 (2004)[arXiv:hep-ph/0406017]; arXiv:hep-ph/0409195; H. Fritzsch and Z. z. Xing, Phys. Lett. B 598, 237 (2004) [arXiv:hep-ph/0406206].

[9] I. Dorsner and A. Y. Smirnov, Nucl. Phys. B 698, 386 (2004) [arXiv:hep-ph/0403305].

[10] A. Yu. Smirnov, [arXiv:hep-ph/0402264]; M. Raidal, Phys. Rev. Lett. 93, 161801 (2004) [arXiv:hep-ph/0404046]; H. Minakata and A. Yu. Smirnov, Phys. Rev. D 70, 073009 (2004)[arXiv:hep-ph/0405088]; P. H. Frampton and R. N. Mohapatra, JHEP 0501, 025 (2005)[arXiv:hep-ph/0407139]; J. Ferrandis and S. Pakvasa, Phys. Rev. D 71, 033004 (2005)[arXiv:hep-ph/0412038]; S. Antusch, S. F. King and R. N. Mohapatra, arXiv:hep-ph/0504007;S. F. King, arXiv:hep-ph/0506297; M. Lindner, M. A. Schmidt and A. Yu. Smirnov, arXiev:hep-ph/0505067. For a review and further references, see, H. Minakata, arXiv:hep-ph/0505262.

[11] C. Giunti and M. Tanimoto, Phys. Rev. D 66, 113006 (2002)[arXiv:hep-ph/0209169]; P. H. Frampton, S. T. Petcov and W. Rodejohann, Nucl. Phys. B 687, 31 (2004)[arXiv:hep-ph/0401206]; A. Datta, L. Everett and P. Ramond, Phys. Lett. B620 42 (2005); S. Antusch, S. F. King, arXiv:hep-ph/0508044; F. Plentinger, W. Rodejohann, Phys.Lett. B625:264 (2005)[ arXiv:hep-ph/0507143].

[12] B. Pontecorvo, Sov. Phys. JETP 6429 (1957)[Zh. Eksp. Teor. Fiz. 33549 (1957)]; Z. Maki, M. Nakagawa and S. Sakata, Prog. Theor. Phys. 28870 (1962).

[13] P. F. Harrison, D.H. Perkins and W.G. Scott, Phys. Lett. B 530167 (2002) [arXiv:hep-ph/0202074].

[14] G. C. Branco, M. N. Rebelo and J. I. Silva-Marcos, Phys. Rev. Lett. 82, 683 (1999) [arXiv:hep-ph/9810328].

[15] M. Maltoni, T. Schwetz, M. A. Tortola and J. W. F. Valle, New J. Phys. 6, 122 (2004) [arXiv:hep-ph/0405172]; A. Strumia and F. Vissani, arXiv:hep-ph/0503246; G. L. Fogli, E. Lisi, A. Marrone, A. Palazzo and A. M. Rotunno, arXiv:hepph/0506307; A. Bandyopadhyay, S. Choubey, S. Goswami, S. T. Petcov and D. P. Roy, Phys. Lett. B 608, 115 (2005) [arXiv:hep-ph/0406328]. 
[16] Letter of Intenet for Double Chooz, F. Ardellier et al, hep-ex/0405032.

[17] http://jkj.tokai.jaeri.go.jp/. 